\title{
LEAN SIX SIGMA E INDUSTRIA 4.0, UNA REVISIÓN DESDE LA ADMINISTRACIÓN DE OPERACIONES PARA LA MEJORA CONTINUA DE LAS ORGANIZACIONES
}

\author{
AUTORES: José Felipe Ramírez Pérez ${ }^{1}$ \\ Virginia Guadalupe López Torres ${ }^{2}$ \\ Sergio Alonso Hernández Castillo ${ }^{3}$ \\ Maylevis Morejón Valdés ${ }^{4}$
}

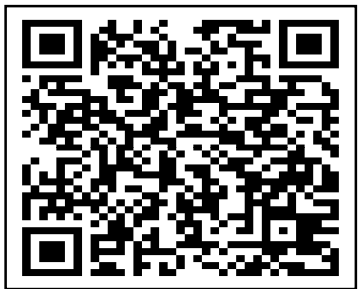

DIRECCIÓN PARA CORRESPONDENCIA: jramirez14@uabc.edu.mx

Fecha de recepción: 12/01/2021

Fecha de aceptación: 20/06/2021

\section{RESUMEN}

La administración de operaciones es un área de estudio de la administración que se centra en la gestión estratégica de las organizaciones. En un contexto mundial marcado por la globalización, la Industria 4.0 e impactado por la pandemia Covid-19, que ha generado mayor incertidumbre y complejidad para las empresas, la adopción de metodologías, métodos y herramientas de administración de operaciones constituye una necesidad para mejorar el desempeño empresarial. El objetivo de la investigación es discutir la contribución actual que tiene la metodología Lean Six Sigma en la mejora continua de las organizaciones, el aumento de la productividad y la competitividad. Se realiza la discusión a partir del análisis de las principales aseveraciones realizadas por académicos, investigadores y empresarios de reconocido prestigio en la temática a nivel mundial. La investigación tiene un enfoque cualitativo, el alcance es exploratorio y el diseño es no experimental. El análisis documental realizado por medio de la plataforma de la Web of Sciences de Clarivate Analytics arrojó 1015 resultados, los cuales fueron procesados por medio de la implementación de la metodología PRISMA. Los resultados obtenidos permiten afirmar que la implementación de la metodología Lean Six Sigma en las organizaciones contribuye a aumentar los niveles de productividad y competitividad. Los trabajos futuros deben dirigirse a evaluar cuantitativamente el impacto real y los beneficios que aporta a las empresas su adopción en el logro de una mayor eficiencia, productividad y obtención de ventajas competitivas sostenidas.

\footnotetext{
${ }^{1}$ Ingeniero en Ciencias Informáticas. Facultad de Ciencias Administrativas y Sociales. Universidad Autónoma de Baja California, Unidad Valle Dorado. Ensenada, Baja California, México. E-mail: jramirez14@uabc.edu.mx

${ }^{2}$ Doctora en Ciencias Administrativas. Facultad de Ciencias Administrativas y Sociales. Universidad Autónoma de Baja California, Unidad Valle Dorado. Ensenada, Baja California, México. E-mail: virginia.lopez@uabc.edu.mx

${ }^{3}$ Máster en Ingeniería. Facultad de Ciencias Administrativas y Sociales. Universidad Autónoma de Baja California, Unidad Valle Dorado. Ensenada, Baja California, México. E-mail: sergio.hernandez@uabc.edu.mx

${ }^{4}$ Ingeniera en Ciencias Informáticas. Facultad de Ciencias Administrativas y Sociales. Universidad Autónoma de Baja California, Unidad Valle Dorado. Ensenada, Baja California, México. E-mail: maylevis.morejon@uabc.edu.mx
} 
José F. Ramírez Pérez, Virginia G. López Torres, Sergio A. Hernández Castillo, Maylevis Morejón Valdés

PALABRAS CLAVE: administración de operaciones; competitividad; Industria 4.0; manufactura esbelta; productividad; seis sigma.

\title{
LEAN SIX SIGMA AND INDUSTRY 4.0, A REVIEW FROM OPERATIONS MANAGEMENT FOR THE CONTINUOUS IMPROVEMENT OF ORGANIZATIONS.
}

\begin{abstract}
Operations management is an area of management study that focuses on the strategic management of organizations. In a world context marked by globalization, Industry 4.0 and impacted by the Covid-19 pandemic, which has generated greater uncertainty and complexity for companies, the adoption of operations management methodologies, methods and tools is a necessity to improve business performance. The objective of the research is to discuss the current contribution of the Lean Six Sigma methodology in the continuous improvement of organizations, the increase of productivity and competitiveness. The discussion is based on the analysis of the main assertions made by academics, researchers and businessmen of recognized prestige in the subject worldwide. The research has a qualitative approach, the scope is exploratory and the design is non-experimental. The documentary analysis carried out through the Clarivate Analytics Web of Sciences platform yielded 1015 results, which were processed through the implementation of the PRISMA methodology. The results obtained allow affirming that the implementation of the Lean Six Sigma methodology in organizations contributes to increase productivity and competitiveness levels. Future work should be directed to quantitatively evaluate the real impact and benefits that its adoption brings to companies in achieving greater efficiency, productivity and obtaining sustained competitive advantages.
\end{abstract}

KEYWORDS: competitiveness; Industry 4.0; Lean Manufacturing, operations management; productivity; Six Sigma.

\section{INTRODUCCIÓN}

Según diversos autores como Chase, Jacobs \& Aguilano (2009), Schroeder \& Olaeta (1992) y Schroeder, Almada \& Santalla (2005), la administración de operaciones se entiende como el conjunto de actividades que se realizan en las organizaciones para la generación de valor en forma de bienes y servicios, como resultado de la transformación de las materias primas en productos terminados. La administración de operaciones se caracteriza por ser una de las tres funciones principales de una organización, siendo las restantes dos: el marketing y la contabilidad (Chase et al., 2009; Schroeder \& Olaeta, 1992; Schroeder et al., 2005). Por todo ello, la administración de operaciones es determinante en el logro de niveles elevados de eficiencia, rendimiento, productividad y competitividad empresarial (Cristo et al., 2020; Pérez et al., 2016).

Actualmente, la administración de operaciones tiene una gran importancia y connotación, en un contexto mundial marcado por la globalización, el progresivo empleo de herramientas tecnológicas, la Industria 4.0 e impactado por la pandemia Covid-19. Este escenario y contexto ha generado una mayor incertidumbre y complejidad para las empresas. Es por eso que la administración de operaciones favorece la gestión estratégica para el logro efectivo de los objetivos con mayor rendimiento, eficiencia, productividad y competitividad (Granda, Manzo, Villamar \& Viteri, 2017). En tal sentido, Damian \& Suárez-Barraza (2015) y Heizer \& Render 
(2004) coinciden en sus estudios que las empresas que aplican técnicas de administración de operaciones obtienen mejores resultados y tienen un mejor desempeño competitivo, en comparación con las empresas que no innovan en operación. Algunas de las técnicas que se aplican en la administración de operaciones van dirigidas al mejoramiento del negocio y de los procesos de producción (Chacón \& Zavaleta, 2014; Mayorga \& Vásquez, 2016; Ramírez, 2016; Schroeder \& Olaeta, 1992).

En el mismo orden de discusión, se puede evaluar la pertinencia de la aplicación efectiva de la administración de operaciones en las organizaciones que prestan servicios como el sector hotelero y la salud. En tal sentido la administración de operaciones permite y propicia el aseguramiento de la calidad de la cobertura de salud que se les brinda a los pacientes, así como el control de los costos y recursos. Adicionalmente, permite la prestación de servicios efectivos, seguros y centrados en el paciente, sin la generación de sobrecostos en áreas claves como la gestión de los inventarios (Atasu et al., 2020; José Felipe \& Raynel, 2015; Mišić \& Perakis, 2020).

Algunas de los beneficios o ventajas que se le atribuyen a la administración de operaciones son la generación de una mayor ventaja competitiva, a partir de un mejor conocimiento de los elementos internos que tiene la organización y los elementos externos con los que interactúa (Manikas, Patel, \& Oghazi, 2019). La identificación de estos aspectos posibilita el conocimiento del entorno operativo para poder adaptar estrategias y herramientas de manera eficiente y rápida cuando se identifican entornos cambiantes y de gran incertidumbre (Lu \& Shen, 2020). Del mismo modo, la reducción de costos constituye uno de sus beneficios principales. La implementación del método JIT (Just In Time, por sus siglas en inglés), aplicado a los sistemas de producción, puede catalogarse como una herramienta muy pertinente para mitigar los gastos, por medio de una correcta gestión de los costos de inventario. Del mismo modo, esta reducción de costos y ahorro de recursos monetarios puede ser posible a partir de la eliminación de gastos innecesarios (Phan, Nguyen, Nguyen \& Matsui, 2019).

En cuanto a la satisfacción del cliente, la administración de operaciones también tiene una incidencia directa, al posibilitar la implementación de sistemas de gestión de la calidad que posibiliten satisfacer las necesidades de los consumidores. De este modo se puede ejercer un mayor control sobre la planificación de los procesos de producción, el control de los inventarios y la capacidad de los empleados, para que todo esto tribute en una mayor satisfacción del usuario final en el producto o servicio que se le brinde (Demir, 2019; Torlak, Demir \& Budur, 2019). Asociado a lo anterior se encuentra la motivación de los empleados, la cual también puede ser posible a través de la correcta gestión de las operaciones, en donde se logra una identificación efectiva de la responsabilidad o función que tiene cada trabajador en la organización. De esta manera, el trabajador no se siente desmotivado, como un ente aislado o excluido de los procesos y decisiones al no saber su rol dentro de la empresa.

El gerente de operaciones, como parte del logro adecuado de sus funciones de planeación, organización, dirección y control, comúnmente ejecuta un conjunto de decisiones estratégicas que pueden considerarse como ventajas en su utilización. Algunas de estas decisiones estratégicas del área de administración de operaciones son: el diseño de los bienes y servicios, la administración de la calidad, la administración de la cadena de suministro, el mantenimiento, la gestión de los 
José F. Ramírez Pérez, Virginia G. López Torres, Sergio A. Hernández Castillo, Maylevis Morejón Valdés

inventarios, la programación, el mantenimiento, el desarrollo de estrategias de procesos, distribución de instalaciones y localizaciones, así como la gestión de los recursos humanos (Heizer \& Render, 2004).

En la última década, como parte de las innovaciones en la administración de operaciones han emergido dos nuevos conceptos: la metodología Seis Sigma (Six Sigma, como se le conoce en inglés) y la Manufactura Esbelta (Lean Manufacturing, como se le conoce en inglés). Las mismas han significado una revolución hacia el interior de la organización, en la obtención de manera más óptima de la mejora de los procesos, productos y servicios, así como en el incremento de la rentabilidad y productividad de estos (Makwana \& Patange, 2021). La adopción de la metodología Lean Six Sigma, como una conjugación de ambos métodos o metodologías, ha propiciado la disminución de la variabilidad de los procesos y la eliminación de los desperdicios, dándole prioridad a las exigencias de los clientes, relacionadas con los requisitos preestablecidos del producto final. Además, esta metodología está orientada o se centra fundamentalmente en las cadenas de suministro y la logística (Mangla et al., 2020; Sindi \& Roe, 2017; Tay \& Loh, 2021).

El objetivo que tiene la investigación es discutir la contribución actual que tiene la metodología Lean Six Sigma en la mejora continua de las organizaciones, el aumento de la productividad y la competitividad. Para ello se realiza desde un enfoque de la administración de operaciones y contextualizado a un entorno marcado por la Industria 4.0. Se realiza la discusión a partir del análisis de las principales aseveraciones realizadas por académicos, investigadores y empresarios de reconocido prestigio en la temática a nivel mundial.

\section{ANTECEDENTES}

Si bien la administración de operaciones tiene sus antecedentes desde que el ser humano ha producido bienes y servicios para satisfacer sus necesidades, sus principales aportes se pueden enmarcar en los últimos 250 años, como establece Monterroso (2014). Es por ello que se realizó un análisis bibliométrico en la Web of Sciences en el periodo comprendido de 2017-2021 con los términos "competitiveness, Industry 4.0, Lean Manufacturing, operations management, productivity y Six Sigma". El propósito del análisis bibliométrico fue caracterizar los principales estudios que se han realizado en el área de conocimiento en cuestión. A continuación, en la Figura 1 se muestran las 1015 investigaciones obtenidas con los términos clave especificados en el periodo de 2012 a 2021 para evaluar la evolución de la temática a nivel global. 


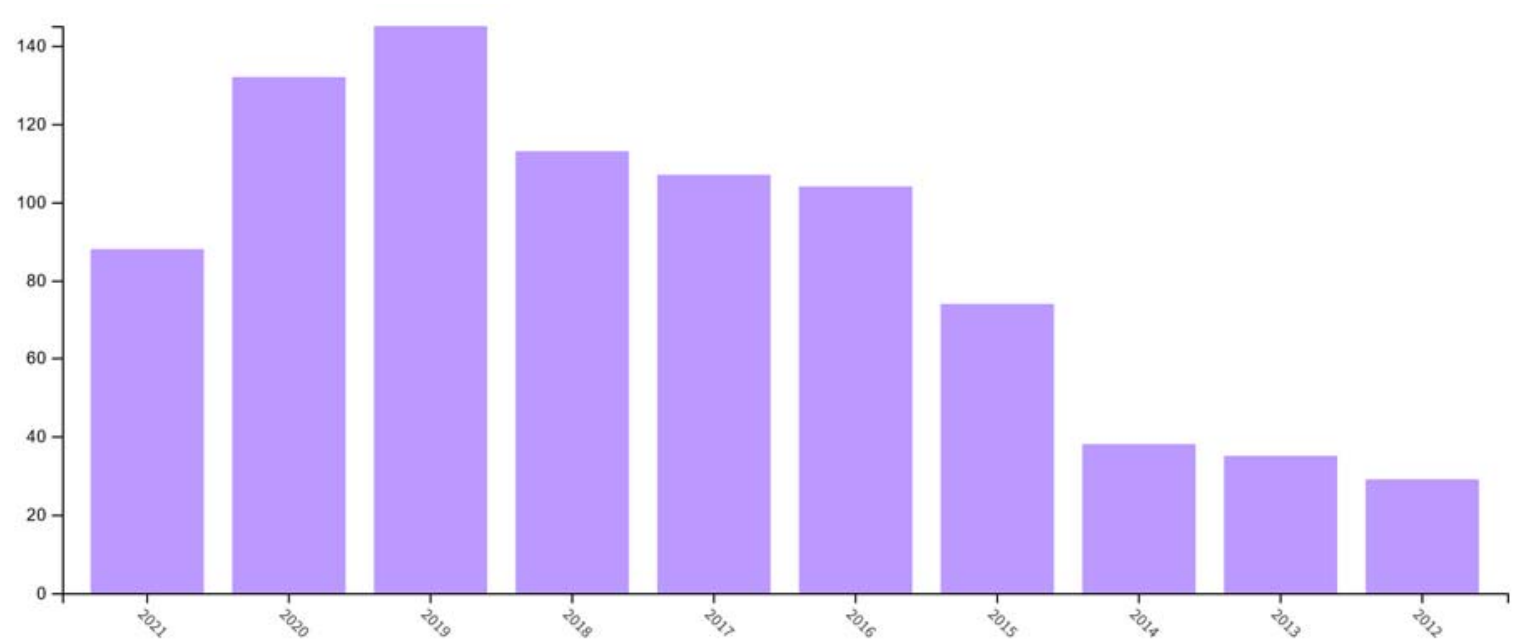

Figura 1. Evolución del tratamiento del Lean Six Sigma en la Web of Sciences para el periodo 2012-2021. Fuente: elaboración propia con datos obtenidos de Clarivate Analytics.

Asimismo, en el análisis realizado se evidencia que las principales áreas de investigación que emplean "competitiveness, Industry 4.0, Lean Manufacturing, operations management, productivity y Six Sigma" son la Administración con un 31.5\% del total de las investigaciones realizadas en el periodo. En segundo lugar destaca la Ingeniería Industrial con el 21.6\%. Luego se visualiza un variado grupo de áreas de investigación, sectores industriales y de los servicios que emplean Lean Six Sigma como son las ciencias de la gestión y la investigación de operaciones, los servicios de ciencias de la salud y políticas de salud y los procesos de fabricación ingenieriles, entre otros. Todo lo anterior permite demostrar la necesidad de profundizar en el análisis de la cuestión. A continuación en la Figura 2 se muestra el análisis del empleo de Lean Six Sigma según el área de conocimiento y sectores de la sociedad donde se ha aplicado: 


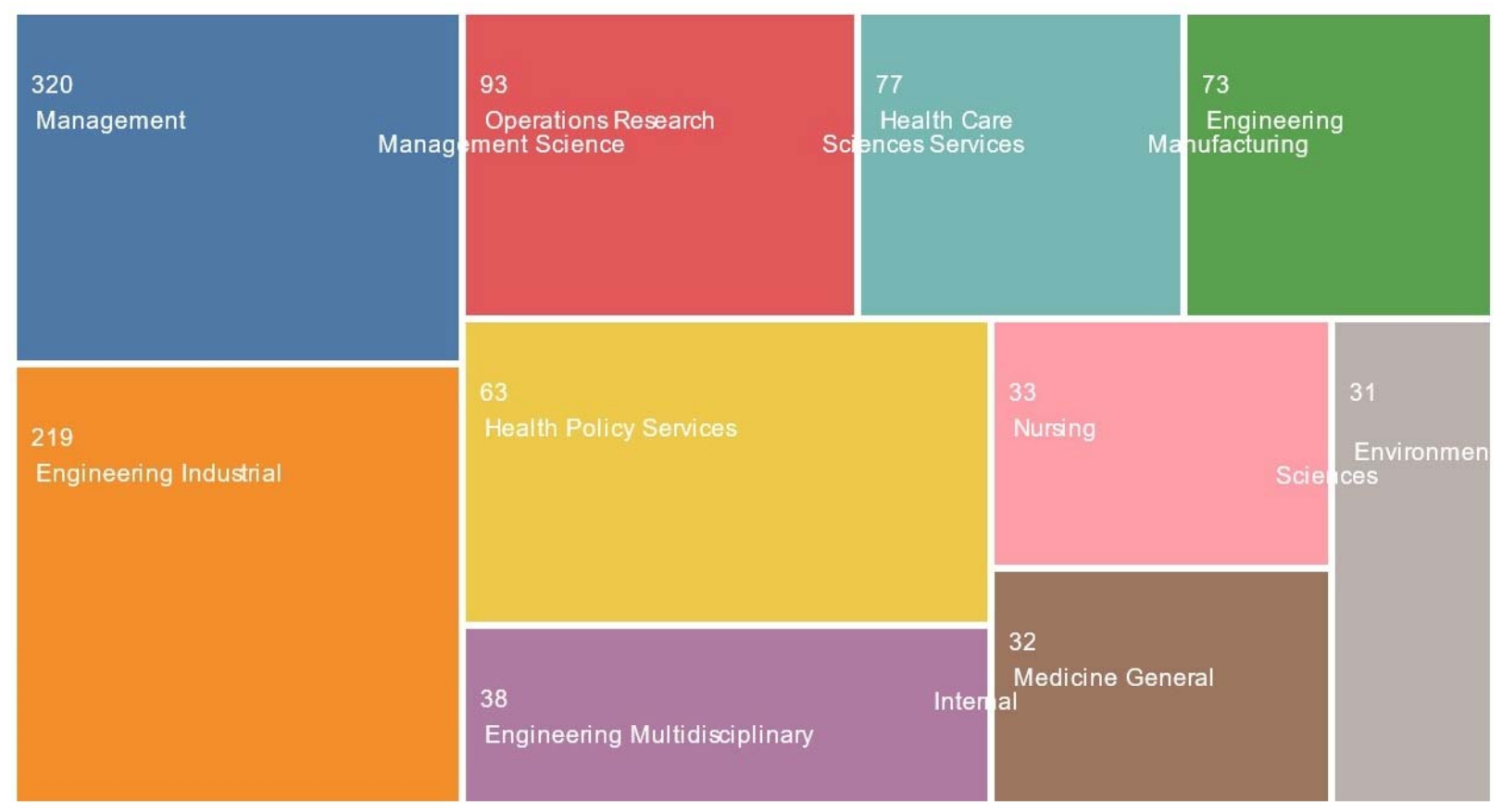

Figura 2. Análisis del empleo de Lean Six Sigma según el área de conocimiento y sectores de la sociedad donde se ha aplicado. Fuente: elaboración propia con datos obtenidos de Clarivate Analytics.

Finalmente, en la Figura 3 se muestran las regiones donde se ha generado el mayor número de innovaciones en materia de administración de operaciones y la aplicación de la metodología Lean Six Sigma para la mejora continua de los procesos de fabricación de bienes y servicios y prestación de servicios de mayor calidad, por ende, las regiones donde hay mayor madurez y desarrollo en este sentido. Del total de invenciones, el 37.73\% es creada por los Estados Unidos de América, siendo la India el segundo lugar con el 14.58\%. Adicionalmente, otros países destacados son Escocia e Inglaterra en Europa. Del mismo modo, llama la atención que de Latinoamérica aparece Brasil con un número significativo de innovaciones en materia de administración de operaciones. 

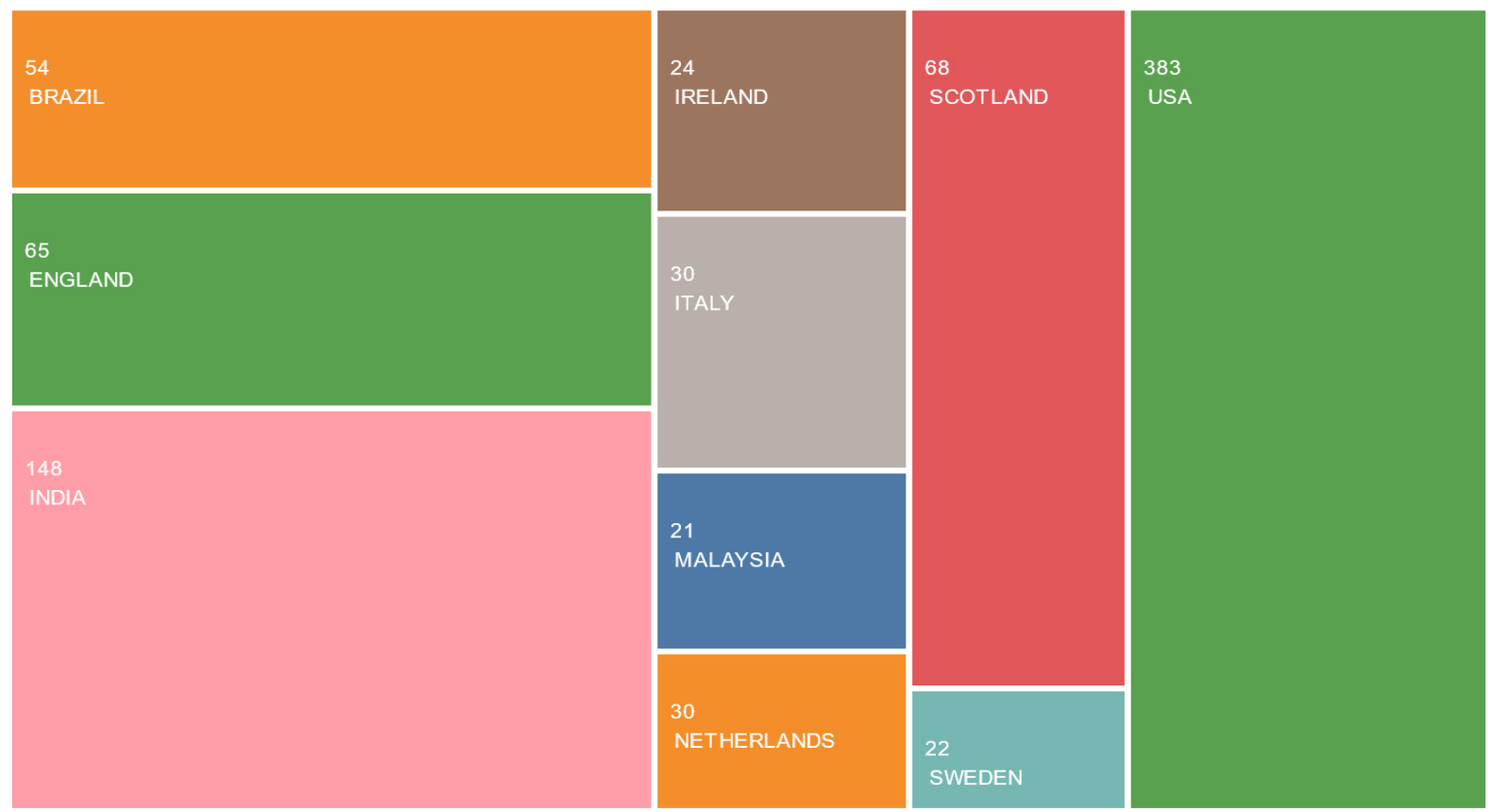

Figura 3. Análisis del empleo de Lean Six Sigma según la región geográfica. Fuente: elaboración propia con datos obtenidos de Clarivate Analytics.

\section{MÉTODO}

El manuscrito tiene el objetivo de discutir la contribución actual que tiene la metodología Lean Six Sigma en la mejora continua de las organizaciones, el aumento de la productividad y la competitividad. Para ello se realiza desde un enfoque de la administración de operaciones y contextualizado a un entorno marcado por la Industria 4.0. Para ello, la discusión se realiza en tres líneas de dirección:

- Metodología Six Sigma como método basado en datos para aumentar la calidad y la disminución de la variabilidad en los procesos de producción, que aseguren la entrega de un producto o servicio al cliente con la menor cantidad de fallos posibles.

- Lean Manufacturing como modelo de gestión para la minimización de las pérdidas en el proceso de producción y maximización de valor para el cliente final, imprimiéndole velocidad a los procesos de producción.

- Metodología Lean Six Sigma para la optimización y mejora continua de procesos, al combinar las mejoras en la calidad obtenidas con Six Sigma, con las mejoras de velocidad obtenidas con Lean Manufacturing.

- Discusión de las aportaciones de Lean Six Sigma en la mejora continua de las organizaciones, el aumento de la productividad y la competitividad. Se realiza la discusión a partir del análisis de las principales aseveraciones realizadas por académicos, investigadores y empresarios de reconocido prestigio en la temática a nivel mundial, con un alto grado de actualidad en el periodo 2017-2021. Se analizaron 586 artículos 
José F. Ramírez Pérez, Virginia G. López Torres, Sergio A. Hernández Castillo, Maylevis Morejón Valdés

indexados en la Web of Sciences Core Collection, estando el 100\% escrito en idioma inglés.

La investigación tiene un enfoque cualitativo, el alcance es exploratorio y el diseño es no experimental. Las palabras clave empleadas para la búsqueda de información, análisis y discusión de resultados son: Administración de operaciones, Competitividad, Industria 4.0, Lean Manufacturing, Productividad y Six Sigma. El propósito del estudio es llevar a cabo una revisión bibliográfica, análisis bibliométrico y discusión preliminar concentrado fundamentalmente entre los años 2017-2021, en donde se evidencie la importancia de la metodología Lean Six Sigma en la mejora continua de las organizaciones. Para este fin se discuten los principales aportes dados por los empresarios e investigadores especializados en la temática, para llegar a conclusiones.

El análisis documental realizado por medio de la plataforma de la Web of Sciences de Clarivate Analytics incluyó la búsqueda en las bases de datos Web of Science Core Collection, SciELO Citation Index, Russian Science Citation Index y KCI-Korean Journal Database. Entre 2012 y 2021 la búsqueda arrojó 1015 resultados de innovación que incluyeron los términos "competitiveness, Industry 4.0, Lean Manufacturing, operations management, productivity y Six Sigma”. Del mismo modo, la búsqueda en el periodo comprendido de 2017 a 2021 arrojó 586 resultados, donde el $61 \%$ de los mismos responde a las áreas de Administración Empresarial e Ingeniería Industrial. Asimismo, el 81.6\% de los documentos corresponden a artículos originales, mientras que el $12 \%$ son artículos de revisión. El 98.5\% del total de documentos son escritos en idioma inglés, donde solo el $31.4 \%$ es de acceso abierto. Por último, con el $31.6 \%$ y $19.1 \%$, los EEUU y la India son los países que mayores contribuciones tiene en el área.

En su ejecución fue empleada la metodología PRISMA, la cual facilita la presentación de los resultados de la revisión sistemáticas de la literatura, aplicada también a los meta-análisis. PRISMA se compone de una lista de verificación de 27 aspectos y de un diagrama de flujo de cuatro fases, el cual se muestra a continuación en la Figura 4. 


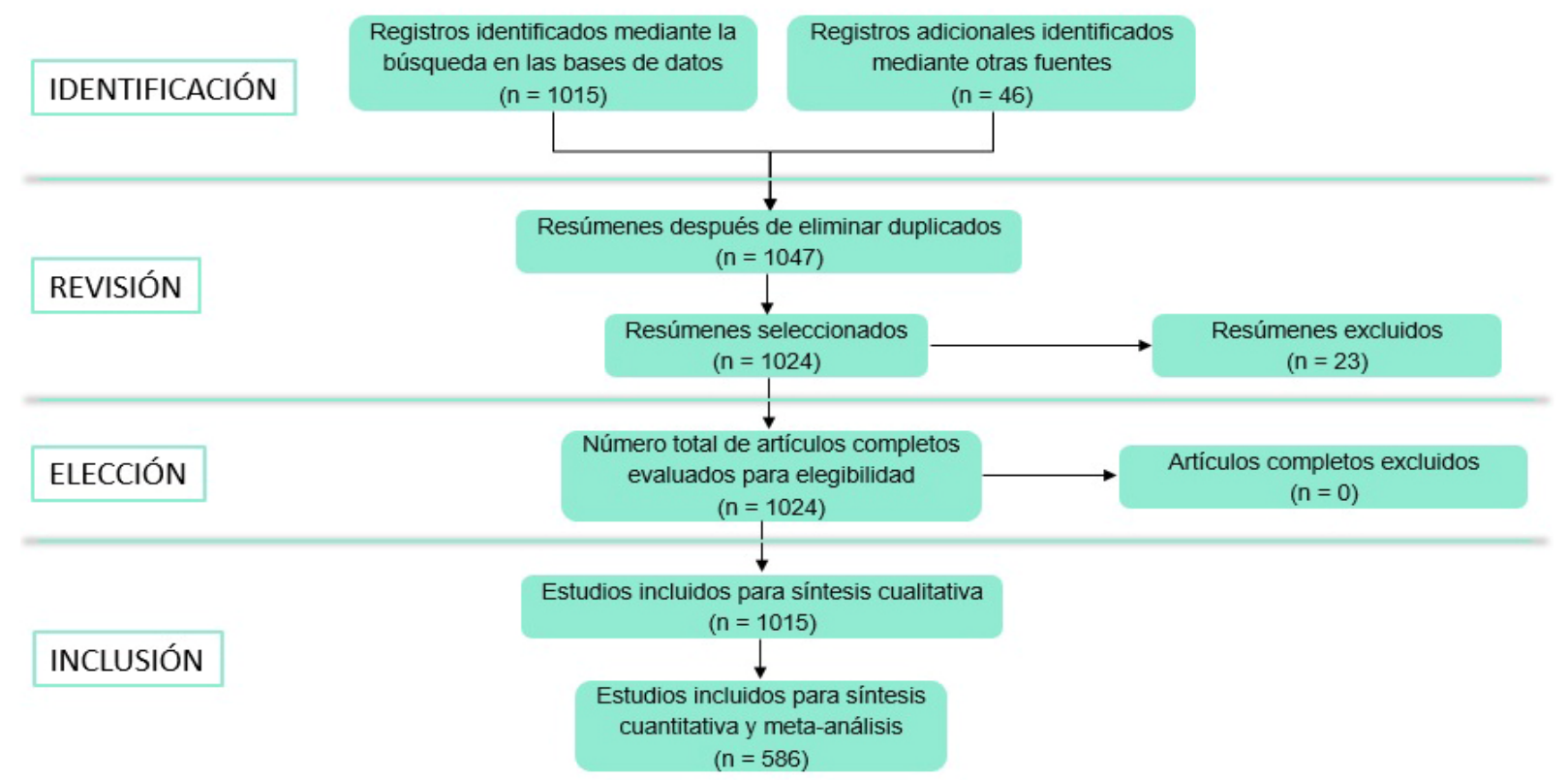

Figura 4. Implementación de la metodología PRISMA en el análisis documental para la discusión de las aportaciones de Lean Six Sigma en la optimización de procesos. Fuente: elaboración propia. 


\section{RESULTADOS Y DISCUSIÓN}

El resultado fundamental de la investigación se centra en el análisis de las metodologías Six Sigma y Lean Manufacturing, y la posterior discusión de la contribución actual que tiene la metodología Lean Six Sigma en la mejora continua de las organizaciones, el aumento de la productividad y la competitividad. Los análisis son realizados desde un enfoque de la administración de operaciones y contextualizado a un entorno marcado por la Industria 4.0. Posteriormente, se realiza la discusión a partir del análisis de las principales aseveraciones realizadas por académicos, investigadores y empresarios de reconocido prestigio en la temática a nivel mundial.

\section{Metodología Six Sigma}

La metodología Six Sigma fue desarrollada por Motorola en 1980. Es un método basado en datos para aumentar la calidad y la disminución de la variabilidad en los procesos de producción, que aseguren la entrega de un producto o servicio al cliente con la menor cantidad de fallos posibles. Six Sigma tiene diez principios, los cuales son (Antony Snee \& Hoerl, 2017):

1. Liderazgo comprometido de arriba hacia abajo, a todos los niveles de la organización.

2. Estructura directiva que incluye personal a tiempo completo.

3. Formación y entrenamiento continuo de cada uno de los actores que intervienen en la utilización del Six Sigma.

4. Acreditación orientada al cliente y enfocada a que los procesos cumplan con los requerimientos del cliente.

5. Dirigida con datos y soportado en un pensamiento estadístico para la mejora de procesos.

6. Metodología robusta.

7. Los proyectos generan ahorros o aumento en ventas.

8. El trabajo se reconoce.

9. Proyectos largos.

10. Comunicación a todos los niveles, dentro y fuera de la organización.

Del mismo modo, el equipo de trabajo de Six Sigma se compone de:

1. Un director Six Sigma, el cual define los objetivos estratégicos, las responsabilidades, selecciona el proyecto y los equipos que formarán parte de él, entre otras actividades.

2. Altos directivos (champions): Son los responsables de cada una de las áreas, quienes proveen la administración estratégica y los recursos para los proyectos a realizarse.

3. Cinturones negros maestros (master black belts): Es un personal con elevada experticia, que ya ha desarrollado actividades de cinturón negro, por lo que dirigen y capacitan a los cinturones negros como expertos Six Sigma.

4. Cinturones negros (black belts): Expertos técnicos que se dedican a la metodología Six Sigma. Lideran los proyectos y mantienen una cultura de mejora de procesos. Son los que capacitan a los cinturones verdes y tienen la obligación de encontrar y proponer mejoras.

5. Cinturones verdes (green belts): Expertos técnicos con dedicación parcial a actividades de Six Sigma. Se encargan de liderar proyectos para atacar problemas de sus áreas.

160 UNESUM-Ciencias. Publicación cuatrimestral. Vol. 5, Año 2021, No. 4 (Número Especial) 


\section{Lean Manufacturing}

Lean Manufacturing es un modelo de gestión para la minimización de las pérdidas en el proceso de producción y maximización de valor para el cliente final, imprimiéndole velocidad y calidad a los procesos de producción (Correa, 2007). Se entiende también como un sistema de organización del trabajo que se centra en la mejora del sistema de producción, por medio de la eliminación de las tareas que no aportan valor al cliente ni al proceso. Fue desarrollado en Toyota en la década de 1970, donde fue definida como una filosofía que se basa en: la eliminación de los desperdicios, el respeto al trabajador y la mejora consistente de la productividad y la calidad.

Lean Manufacturing se centra en la reducción de los ocho tipos de desperdicios (Correa, 2007):

1. Sobreproducción

2. Tiempo de espera

3. Transporte

4. Exceso de procedimientos

5. Inventario

6. Movimientos

7. Defectos

8. No utilizar la creatividad de la gente

De igual manera, Lean Manufacturing presenta siete principios, los cuales son (Correa, 2007):

1. Hacerlo bien desde el inicio, con cero defectos.

2. Excluir actividades que no añaden valor al cliente.

3. La mejora continua, donde se eleva la calidad de los productos y servicios, y la productividad, al mismo tiempo que se reducen los costos.

4. Procesos pull, basados en la existencia de una demanda, evitando la existencia de stocks.

5. Flexibilidad en la diversificación de productos, con un ajuste exacto de las cantidades.

6. Colaborar con los proveedores, por medio de la construcción de relaciones duraderas, con acuerdos donde se comparten costos y riesgos.

7. Cambio del enfoque de ventas: el cliente adquiere una solución, no un producto o servicio.

La operatividad de cada uno de los principios se logra con la implantación de estrategias como Kanban, JIT, TPM y $5 \mathrm{~S}$. Particularmente, la aplicación de las $5 \mathrm{~S}$ tiene muchos beneficios para la organización pues fomenta el trabajo en equipo, el sentido de pertenencia, la creación de un clima organizacional favorable, el aprovechamiento del tiempo y la motivación de los trabajadores (Singh, Pradhan, Ravi \& Dhale, 2020). Adicionalmente, se reducen las mermas y los desperdicios, y se elaboran productos de mayor calidad. Las $5 \mathrm{~S}$ se originan de las palabras japonesas:

- seiri: subordinar, clasificar, descartar

- seiton: sistematizar, ordenar

- seiso: sanear y limpiar 
- seiketsu: simplificar, estandarizar y volver coherente

- shitsuke: sostener el proceso, disciplinar

\section{Metodología Lean Six Sigma}

La metodología Lean Six Sigma nace de la complementación de Six Sigma y Lean Manufacturing para la optimización y mejora continua de los procesos organizacionales, teniendo como propósito alcanzar la excelencia operacional (Laureani \& Antony, 2019; Patel \& Patel, 2021). La utilización conjunta de estas metodologías posibilita obtener dobles beneficios por parte de las empresas, donde se combinan las mejoras en la calidad y la disminución de la variabilidad obtenidas con Six Sigma, con las mejoras de velocidad y disminución de desperdicios obtenidas con Lean Manufacturing (da Silva, Filho, Agostinho \& Junior, 2019).

Para su adecuada implementación, Lean Six Sigma utiliza la metodología DMAIC (del inglés, Define, Measure, Analyze, Improve y Control), la cual define seis etapas o pasos:

- Definir: implica el establecimiento detallado de la situación actual de la organización, por medio de los procesos existentes. Se definen los procesos a ser evaluados tal como se ejecutan en la realidad, no como son establecidos en los manuales.

- Medir: incluye la medición y registro de todos los datos existentes para que sea posible la evaluación de la efectividad de los procesos. Esta etapa se basa en un precepto de Peter Druker, que establece que lo que no se puede medir, no se puede mejorar.

- Analizar: se examinan de manera detallada cada una de las actividades del proceso, basado en las estadísticas obtenidas de la etapa anterior, de manera que se puedan distinguir las actividades que aportan valor añadido, para poder arribar a conclusiones.

- Mejorar: dadas las actividades que aportan valor añadido a la organización, la próxima tarea es potenciarlas para ser más eficientes. Para ello, se procede a la reducción y/o eliminación de los desperdicios o desechos que no aportan valor añadido, tales como los defectos, las esperas, la sobreproducción, el talento no empleado y el inventario.

- Controlar: abarca el control y la monitorización constante de los resultados, para mantenerlos y mejorarlos en el tiempo, única vía posible para lograr la mejora continua.

Adicionalmente, para que un proceso sea catalogado como Lean Six Sigma, los fallos de un proceso deben ser como máximo 3,4 Defectos Por cada Millón de Oportunidades (DPMO). De esta manera se pueden clasificar cada uno de los procesos de acuerdo con el nivel de calidad o nivel de DPMO. Para ello, se le asigna un nivel de Sigma variable entre Cero y Seis, dependiendo de los defectos obtenidos, como se muestra en la siguiente tabla:

Tabla 1. Eficiencia de un proceso de acuerdo a sigma. Fuente: Albert, Soler \& Molina (2017).

\begin{tabular}{|c|c|}
\hline Sigma & Eficiencia \\
\hline 1 & $68,27 \%$ \\
\hline 2 & $95,45 \%$ \\
\hline 3 & $99,73 \%$ \\
\hline 4 & $99,994 \%$ \\
\hline 5 & $99,99994 \%$ \\
\hline 6 & $99,999997 \%$ \\
\hline
\end{tabular}


Existen diversas herramientas que se pueden utilizar para aplicar la metodología. Algunas de las más empleadas son: SIPOC (Supplier-Inputs-Process-Outputs-Customers), la matriz causaefecto, la gráfica de Pareto, el diagrama Ishikawa, 5 Why, los análisis de regresión, las pruebas de hipótesis y el Índice de Capacidad del Proceso (PCA), entre otros.

\section{Discusión de las aportaciones de Lean Six Sigma en la optimización de procesos}

En la misma línea de discusión, diversos son los autores que han abordado de las aportaciones de Lean Six Sigma en la optimización de procesos para mejorar la productividad y la competitividad empresarial. A continuación se realiza la discusión a partir del análisis de las principales aseveraciones realizadas por académicos, investigadores y empresarios de reconocido prestigio en la temática a nivel mundial. Para la discusión solo te tuvo en cuenta artículos indexados en la Web of Sciences Core Collection en el periodo de 2017 a 2021.

De acuerdo con da Silva et al. (2019), las empresas basan sus esfuerzos en lograr una competitividad cada vez mayor a través de la productividad y la calidad. En tal sentido los autores consideran que este objetivo es posible por medio de la implementación de la metodología Lean Six Sigma, donde Lean Manufacturing agrega valor al reducir el desperdicio, mientras que Six Sigma reduce la existencia de variabilidad en los productos y servicios ofertados. En su investigación se aplicó un estudio de caso en una empresa manufacturera en Brasil, en donde los resultados de la aplicación de esta metodología propiciaron una mejora en el clima y la cultura organizacional, así como beneficios significativos asociados con la calidad y la productividad. Con tales resultados concuerdan Laureani \& Antony (2017), quienes consideran que el éxito en las implementaciones de Lean Six Sigma tiene una fuerte relación con el liderazgo, en cuanto al aumento del clima organizacional. Dada su hipótesis, los autores identificaron la existencia de diez características de liderazgo más conducentes al éxito en las implementaciones de Lean Six Sigma, entre las que se destacan: la comunicación, la coherencia, la ejemplaridad, la flexibilidad, la percepción de Lean Six Sigma como una filosofía y las capacidades de construir, así como de definir claramente los roles y responsabilidades del equipo.

Jordan, Kušar, Rihar \& Berlec (2019) en cambio abordan directamente las mejoras en el sector empresarial que produce la aplicación de Lean Six Sigma en las dimensiones operativa y de calidad. Los investigadores aseveran que para lograr los requerimientos del cliente en cuanto a plazos de entrega cortos y productos de alta calidad, las empresas tienen necesariamente que aplicar metodologías Lean Six Sigma. Tal conclusión fue posible a través de la realización de varios análisis de flujo de valor, en el que se mostró el tamaño del tiempo de entrega, de la eficiencia del proceso, de la efectividad general del equipo y de la eficiencia del proceso, tanto en empresas organizadas bajo un esquema de producción de forma clásica y otras bajo un esquema de producción Lean Six Sigma. Posteriormente, esta hipótesis es demostrada a partir de un ejemplo de la producción de cubiertas de refrigeración.

Sobre los factores críticos de éxito en la aplicación de Lean Six Sigma en las pequeñas y medianas empresas (PYMEs) aborda la investigación realizada por Stankalla, Koval \& Chromjakova (2018). Según los autores, esta metodología fue aplicada inicialmente dentro de las grandes corporaciones, sin embargo, el interés de las PYMEs en su adopción está aumentando paulatinamente. En tal sentido, afirman que el tamaño de las mismas y la disponibilidad de los 
recursos constituyen un obstáculo para su adecuada implementación. Es por ello que establecen que el compromiso de la alta dirección y la vinculación de Lean Six Sigma con la estrategia empresarial son los factores críticos de éxito de máxima prioridad a tener en cuenta. Del mismo modo, consideran necesario el desarrollo de un buen plan de comunicación y la vinculación de Lean Six Sigma con los clientes para lograr el éxito en su implementación.

En otro orden de discusión, Lee, McFadden \& Gowen (2018), realizaron un análisis exploratorio de la implementación de las metodologías Lean Manufacturing y Six Sigma en los hospitales, dada la poca evidencia empírica de su empleo en el sector de la salud, aun cuando existe un creciente interés en su uso. El estudio tuvo el objetivo de explorar los patrones existentes de implementación de ambas metodologías en hospitales de EEUU, para luego comparar el desempeño de los mismos. Los resultados, soportados en pruebas estadísticas mediante el análisis de conglomerados, contribuyen a la literatura sobre gestión de la calidad al respaldar la relación complementaria entre Lean Manufacturing y Six Sigma en los hospitales. Es por ello que en las conclusiones animan a los profesionales y gerentes en salud a adoptar la implementación de Lean Six Sigma para obtener mejores resultados. A estos resultados finales en el sector de la salud también arriban Henrique \& Filho (2020).

También en el sector de la salud, Trakulsunti, Antony, Edgeman, Cudney, Dempsey \& Brennan (2021) realizaron una investigación en Tailandia, donde evidenciaron cómo con la aplicación de Lean Six Sigma se pudo reducir los errores de dispensación mensuales de 29 incidentes a 6 incidentes en farmacias para pacientes hospitalizados en un hospital público. Ello fue posible por medio de la aplicación exitosa de múltiples herramientas como diagramas de causa y efecto, el análisis de 5 Why y pruebas de hipótesis. Por todo ello, se considera igualmente que Lean Six Sigma aporta numerosos beneficios en la prevención y reducción de errores de medicación.

Por otro lado, Cudney, Venuthurumilli, Materla \& Antony (2020) abordan la aplicación efectiva de esta metodología en un sector distinto al productivo, o en área de investigación no asociadas con la administración empresarial o la ingeniería industrial, en este caso en la educación superior. Los autores refieren que el papel sistemático que juegan los sistemas de mejora continua Lean y Six Sigma también impactan en la calidad de la educación superior. La revisión sistemática realizada por los autores de la literatura relevante sobre ambas metodologías en la educación superior sugirió que estos enfoques se pueden aplicar para mejorar los métodos de enseñanza, los procesos administrativos y otras facetas de las instituciones de educación superior, propiciando una mayor satisfacción al cliente final, en este caso el estudiante.

Asimismo, otros autores han explorado una línea novedosa no abordada directamente con anterioridad, pero que es tan importante como el aumento de la calidad de las producciones y los servicios, la disminución de la variabilidad y de los errores, y la satisfacción de los clientes. Se hace referencia a la huella sobre el planeta. En este sentido, Chugani, Kumar, Garza-Reyes, Rocha-Lona \& Upadhyay (2017) en un estudio exploratorio realizado, por la poca evidencia científica existente al respecto, afirman que la aplicación de Lean Six Sigma puede considerarse un método efectivo para apoyar la conservación de recursos, combatir el calentamiento global y ahorrar energía. En su investigación se muestra que las organizaciones pueden considerar la adopción de Lean Six Sigma para cumplir con las regulaciones ambientales, ahorrar costos y también cumplir con los estándares de gestión de calidad. Esto contribuiría a ayudar a las organizaciones a formular estrategias más efectivas e inclusivas que no solo consideren las dimensiones operativa y de calidad, sino también la dimensión ambiental.

164 UNESUM-Ciencias. Publicación cuatrimestral. Vol. 5, Año 2021, No. 4 (Número Especial) 


\section{CONCLUSIONES}

La administración de operaciones es un área de estudio de la administración, la cual favorece la gestión estratégica de las organizaciones, ya que se encarga de la planificación, organización, dirección, control y mejora de los sistemas de producción de bienes y servicios. Por todo ello, se puede afirmar que la administración de operaciones posibilita la obtención de mayores niveles de eficiencia, productividad y competitividad empresarial, al mismo tiempo que favorece la reducción de errores y costos. Del mismo modo, incide en todas las áreas de la organización, contribuyendo con la mejora continua y sostenida de los procesos estratégicos, operativos y de soporte.

La administración de operaciones guarda una estrecha relación con la eficiencia, el rendimiento, la productividad y la competitividad empresarial. En el contexto nacional actual que vive México, marcado por la globalización, la existencia de la Cuarta Revolución Industrial, la creación de nuevos procesos y modelos de producción, así como el impacto producido por la pandemia COVID-19, una adecuada gestión de las operaciones o de la producción es esencial en la recuperación de la economía, en una mayor inversión extranjera y en una mayor productividad. Ello se debe a que posibilitaría aumentar las capacidades tecnológicas, de innovación, de investigación y de desarrollo.

La metodología Lean Six Sigma posibilita la mejora continua de los procesos, para aumentar el rendimiento, la productividad y la rentabilidad empresarial. Surge de la complementación de la metodología Six Sigma con Lean Manufacturing, en el logro de un esfuerzo conjunto para la reducción de la variación de los procesos y de los residuos. Asimismo, se enfoca en el aumento de la calidad del proceso y de la satisfacción de clientes y empleados. Por todo ello, se puede afirmar que su implementación en el sector empresarial contribuye a aumentar los niveles de productividad y competitividad.

De acuerdo con los análisis realizados y la evidencia científica existente, los trabajos futuros relacionados con la aplicación de la metodología Lean Six Sigma para la mejora continua de las organizaciones, en un escenario marcado por la Industria 4.0, deben dirigirse ante todo a la concientización de gerentes y empleados de que su adopción pasa necesariamente por la existencia de un elevado empoderamiento y compromiso organizacional. Este constituye el primer paso para poder garantizar su implementación efectiva. De la misma forma, se debe llevar a cabo un mayor número de actividades de capacitación y entrenamiento para comprender de manera más adecuada su correcto funcionamiento. Por último, su ejecución eficiente implicará además de una acertada aplicación de la metodología Lean Six Sigma con la administración estratégica de la organización, que permita alienar la mejora prevista de los procesos con las metas y los objetivos que tiene la empresa.

En el mismo orden de discusión, para trabajos futuros se deben desarrollar estudios descriptivos y explicativos, con diseño experimental que evalúen cuantitativamente el impacto real y los beneficios que le brindan a la empresa en México la adopción de la metodología Lean Six Sigma en el logro de una mayor eficiencia, productividad, desempeño competitivo y ventajas competitivas sostenidas. 
José F. Ramírez Pérez, Virginia G. López Torres, Sergio A. Hernández Castillo, Maylevis Morejón Valdés

\section{REFERENCIAS BIBLIOGRÁFICAS}

Albert, E. N., Soler, V. G., \& Molina, A. I. P. (2017). Metodología e implementación de Six Sigma. 3C Empresa, Edición especial. 73-80.

Antony, J., Snee, R., \& Hoerl, R. (2017). Lean Six Sigma: yesterday, today and tomorrow. International Journal of Quality \& Reliability Management.

Atasu, A., Corbett, C. J., Huang, X., \& Toktay, L. B. (2020). Sustainable operations management through the perspective of manufacturing \& service operations management. Manufacturing \& service operations management, 22(1), 146-157.

Chase, R. B., Jacobs, F. R., \& Aquilano, N. J. (2009). Administración de operaciones. Producción y cadena de suministros. Duodécima edición. Mc Graw Hill, México.

Chugani, N., Kumar, V., Garza-Reyes, J. A., Rocha-Lona, L., \& Upadhyay, A. (2017). Investigating the green impact of Lean, Six Sigma and Lean Six Sigma: A systematic literature review. International Journal of Lean Six Sigma.

Correa, F. G. (2007). Manufactura esbelta (lean manufacturing). Principales herramientas. Revista Raites, 1(2), 85112 .

Cristo, N. M., Pérez, J. F. R., \& Izaguirre, L. V. (2020). Estrategia de entrenamiento y acompañamiento a usuarios para el Sistema de Información Hospitalaria XAVIA HIS. Revista Cubana de Informática Médica, 12(1), 76-91.

Cudney, E. A., Venuthurumilli, S. S. J., Materla, T., \& Antony, J. (2020). Systematic review of Lean and Six Sigma approaches in higher education. Total Quality Management \& Business Excellence, 31(3-4), 231-244.

da Silva, I. B., Filho, M. G., Agostinho, O. L., \& Junior, O. F. L. (2019). A new Lean Six Sigma framework for improving competitiveness. Acta Scientiarum. Technology, 41, e37327-e37327.

Damian, I. E., \& Suárez-Barraza, M. F. (2015). Innovación de procesos en la gestión turística: Una revisión de la literatura. Intangible Capital, 11(1), 147-165.

Demir, A. (2019). The impact of strategic operations management decisions on shoppers'wellbeing. Asian Academy of Management Journal, 24(1).

Granda, L. S., Manzo, O. P., Villamar, I. B., \& Viteri, J. V. (2017). Uso de la Administración de Operaciones en las Mipymes. Revista Científica y Tecnológica UPSE, 4(1), 181-187.

Heizer, J., \& Render, B. (2004). Principios de administración de operaciones. Quinta edición. Pearson Educación, México.

Henrique, D. B., \& Filho, M. G. (2020). A systematic literature review of empirical research in Lean and Six Sigma in healthcare. Total Quality Management \& Business Excellence, 31(3-4), 429-449.

Jordan, E., Kušar, J., Rihar, L., \& Berlec, T. (2019). Portfolio analysis of a Lean Six Sigma production process. Central European Journal of Operations Research, 27(3), 797-813.

José Felipe, R. P., \& Raynel, B. T. (2015). Propuesta de Red Cubana Aurora para la colaboración médica a través de Infomed utilizando un enfoque de redes sociales. La Habana: Memorias Convención Internacional de Salud, Cuba Salud.

Laureani, A., \& Antony, J. (2017). Leadership characteristics for lean six sigma. Total Quality Management \& Business Excellence, 28(3-4), 405-426.

Laureani, A., \& Antony, J. (2019). Leadership and Lean Six Sigma: a systematic literature review. Total Quality Management \& Business Excellence, 30(1-2), 53-81.

166 UNESUM-Ciencias. Publicación cuatrimestral. Vol. 5, Año 2021, No. 4 (Número Especial) 
Lee, J. Y., McFadden, K. L., \& Gowen, C. R. (2018). An exploratory analysis for Lean and Six Sigma implementation in hospitals: Together is better?. Health care management review, 43(3), 182-192.

Lu, M., \& Shen, Z. J. M. (2020). A Review of Robust Operations Management under Model Uncertainty. Production and Operations Management.

Makwana, A. D., \& Patange, G. S. (2021). A methodical literature review on application of Lean \& Six Sigma in various industries. Australian Journal of Mechanical Engineering, 19(1), 107-121.

Mangla, S. K., Kusi-Sarpong, S., Luthra, S., Bai, C., Jakhar, S. K., \& Khan, S. A. (2020). Operational excellence for improving sustainable supply chain performance. Resources, Conservation, and Recycling, 162, 105025.

Manikas, A. S., Patel, P. C., \& Oghazi, P. (2019). Dynamic capital asset accumulation and value of intangible assets: An operations management perspective. Journal of Business Research, 103, 119-129.

Mayorga, M. J., \& Vásquez, V. E. (2016). Organización: El rol estratégico de la administración de las operaciones, estratégica de operaciones y su excelencia operacional mediante la innovación y el mejoramiento continuo en los procesos (Disertación doctoral). Universidad Nacional Autónoma de Nicaragua, Managua.

Mišić, V. V., \& Perakis, G. (2020). Data analytics in operations management: A review. Manufacturing \& Service Operations Management, 22(1), 158-169.

Monterroso, E. (2014). El rol estratégico de la Administración de las operaciones en la nueva economía y en el nuevo paradigma de gestión. Revista del Departamento de Ciencias Sociales, 6, 91-135.

Patel, A. S., \& Patel, K. M. (2021). Critical review of literature on Lean Six Sigma methodology. International Journal of Lean Six Sigma.

Pérez, J. F. R., Vázquez, M. L., Valdes, M. M., \& Fajardo, D. O. (2016). Modelo computacional para la recomendación de equipos de trabajo quirúrgico combinando técnicas de inteligencia organizacional. Revista Cubana de Ciencias Informáticas, 10(4), 28-42.

Phan, A. C., Nguyen, H. T., Nguyen, H. A., \& Matsui, Y. (2019). Effect of total quality management practices and JIT production practices on flexibility performance: Empirical evidence from international manufacturing plants. Sustainability, 11(11), 3093.

Ramírez Pérez, J. F. (2016). Modelo para la selección de equipos de trabajo quirúrgico en sistemas de información en salud aplicando técnicas de Inteligencia Organizacional. Disertación doctoral. Universidad de las Ciencias Informáticas, La Habana, Cuba.

Schroeder, R. G., \& Olaeta, R. D. L. P. (1992). Administración de operaciones (Vol. 17). México: McGraw-hill.

Schroeder, R. G., Almada, M. G. C., \& Santalla, J. R. (2005). Administración de operaciones: casos y conceptos contemporáneos. McGraw-Hill.

Sindi, S., \& Roe, M. (2017). The evolution of supply chains and logistics. In Strategic supply chain management (pp. 7-25). Palgrave Macmillan, Cham.

Singh, A., Pradhan, S., Ravi, P., \& Dhale, S. (2020). Application of six sigma and 5 S to improve medication turnaround time. International Journal of Healthcare Management, 1-9.

Stankalla, R., Koval, O., \& Chromjakova, F. (2018). A review of critical success factors for the successful implementation of Lean Six Sigma and Six Sigma in manufacturing small and medium sized enterprises. Quality Engineering, 30(3), 453-468.

Tay, H. L., \& Loh, H. S. (2021). Digital transformations and supply chain management: a Lean Six Sigma perspective. Journal of Asia Business Studies. 
José F. Ramírez Pérez, Virginia G. López Torres, Sergio A. Hernández Castillo, Maylevis Morejón Valdés

Torlak, N. G., Demir, A., \& Budur, T. (2019). Impact of operations management strategies on customer satisfaction and behavioral intentions at café-restaurants. International Journal of Productivity and Performance Management.

Trakulsunti, Y., Antony, J., Edgeman, R., Cudney, B., Dempsey, M., \& Brennan, A. (2021). Reducing pharmacy medication errors using Lean Six Sigma: A Thai hospital case study. Total Quality Management \& Business Excellence, 1-19. 